\title{
No specific relationship between hypnotic suggestibility and the rubber hand illusion: a commentary with new and verification analyses
}

\author{
H. Henrik Ehrsson ${ }^{1^{*}}$ \\ Aikaterini Fotopoulou ${ }^{2}$ \\ Dominika Radziun ${ }^{1}$ \\ Matthew R. Longo ${ }^{3}$ \\ Manos Tsakiris ${ }^{4,5,6^{*}}$ \\ ${ }^{1}$ Department of Neuroscience, Karolinska Institutet, Stockholm, Sweden \\ ${ }^{2}$ Clinical, Educational and Health Psychology Research Department, University College \\ London, UK \\ ${ }^{3}$ Department of Psychological Sciences, Birkbeck, University of London, UK \\ ${ }^{4}$ The Warburg Institute, School of Advanced Study, University of London, UK \\ ${ }^{5}$ Department of Psychology, Royal Holloway, University of London, UK \\ ${ }^{6}$ Department of Behavioural and Cognitive Sciences, Faculty of Humanities, Education and \\ Social Sciences, University of Luxembourg, Luxembourg \\ *Email: Henrik.Ehrsson@ki.se; Manos.Tsakiris@rhul.ac.uk
}

In a recent study, Lush et al. claimed that they found "substantial relationships" (p. 1) between hypnotizability and experimental measures of the rubber hand illusion (RHI) [1]. The authors proposed that hypnotizable participants control their phenomenology to meet task expectations arising from the experimental paradigm. They further suggest that the RHI "may or may not" (p. 5) be entirely explained by hypnotic suggestions driven by task expectancies and therefore could reflect top-down control of perception, instead of multisensory mechanisms. However, in reanalyzing their data, we observe no significant relationships between hypnotic suggestibility and the RHI when quantified using a control condition in line with the authors' preregistered hypothesis. Furthermore, we note that the relationships that the authors describe are weak and observed for a visual "hallucination" control experience and in the control condition, indicating a general influence of hypnotizability on cognition, rather than sensations that specifically relate to the RHI. Overall, the results fit well with the view that the $\mathrm{RHI}$ is a perceptual illusion driven primarily by multisensory mechanisms. 
During the RHI, participants experience a fake hand as their own [1]. In the classic version, synchronous versus asynchronous brushstrokes are applied to the rubber hand in full view of the participant and to the participant's real hand, which is hidden from sight; the synchronous mode of stimulation elicits the illusion of sensing touch on the rubber hand and feeling that the fake hand is one's own. Asynchronous stroking abolishes or significantly reduces the illusion, which is a commonly used control condition [1-3]. Although different theoretical models of the RHI have been proposed [4-6], there is substantial agreement that the RHI is a perceptual illusion that arises from a combination of bottom-up and top-down processes involved in multisensory integration.

In contrast with this view, Lush and colleagues [7] concluded that the RHI arises from hypnotic suggestibility at least partially, and they claimed that trait phenomenological control predicts the RHI. A total of 353 participants were tested on the RHI using the synchronous (illusion) and asynchronous (control) conditions. One-third of the participants were told that they would experience a stronger illusion in the synchronous conditions, another third were told that the effect would be stronger in the asynchronous condition, and the remaining participants were not given instructions. According to the authors' predictions, these different suggestion instructions should influence expectations and the RHI, but this manipulation failed (see further below). The RHI was assessed using a standard questionnaire and a 'proprioceptive drift test' that assessed the change in hand position sensed toward the rubber hand. Individual differences in hypnotic suggestibility were quantified using the SussexWaterloo Scale of Hypnotizability (SWASH)[7]. Importantly, when contrasting the synchronous and asynchronous conditions in the group that received no suggestion instructions, in line with the preregistered hypothesis, no evidence for a relationship between the SWASH scale and the illusion ratings was observed, and only anecdotal evidence in favor of a relationship with proprioceptive drift was shown (nonsignificant two-tailed, $p=0.074$ ) [7]. We reanalyzed the entire sample to maximize the power and observed nonsignificant relationships for both illusion ratings (Fig $1 \mathrm{~A} ; \mathrm{p}=0.2187$ ) and for proprioceptive drift (Fig 1B; $p=0.3758$ ). Moreover, a Bayesian analysis indicated that, for the illusion ratings, the null hypothesis was 5.68 times more likely than the alternative hypothesis (Fig 1A); and when analyzing the illusion statement individually we observed even stronger evidence against the pre-registered hypothesis (Suppl Fig 1A-C). Furthermore, when dividing the participants into four quartiles based on their SWASH scores, we observed that all four groups showed significant differences $(p<0.05)$ between the synchronous and asynchronous conditions on the illusion questions (Fig 1C). Furthermore, all four groups affirmed the illusion experience in the synchronous condition in that their ratings were significantly greater than zero $(p<0.05$; Fig 1C). Thus, even the least suggestible participants showed clear evidence of experiencing the illusion.

Following the unsuccessful attempt to manipulate RHI expectations by explicit suggestion and inconclusive results in preregistered analyses, Lush and colleagues used the whole sample to examine post hoc, exploratory correlations between the SWASH score and the two illusion measures when considering the synchronous condition in isolation. However, the hypnotic suggestibility trait accounted for only approximately $9 \%$ of the illusion ratings' variability. A similar relationship was found for the asynchronous control condition (9\%) and a control statement describing an 'impossible' experience of visual hallucinations (12\%). Noteworthy, when subtracting the illusion statement ratings from the control ratings no positive 
relationship with SWASH was observed, (Suppl Fig 1D-F). Thus, hypnotizability seems to influence cognition and experience rather broadly, but it does not drive the perceptions that specifically relate to the illusion condition, i.e., the sensing of touches on the synchronously stroked rubber hand and the condition-specific increase in the feeling of fake hand ownership. Moreover, due to the nonspecificity of the post hoc findings, it cannot be excluded that they relate to different types of cognitive biases, including behavioral compliance or response bias, in addition to 'genuine' phenomenological control effects.

The authors' argument for downplaying the comparison of experimental conditions was that this approach is supposedly not motivated by the literature. In support of this allegation, they cited the 20 most cited publications on the $\mathrm{RHI}$ using questionnaires or proprioceptive drift measures, respectively, resulting in a list of 30 studies in total (Supplementary information [7]). However, their list: (1) referred to different illusion paradigms; (2) was small in comparison to the relevant $\mathrm{RHI}$ studies that operationalized the illusion effect as differences between conditions; and (3) was not supported by recent reviews on the RHI that emphasized the importance of using control conditions $[3,5]$. Moreover, and contrary to their claims, 28 of the 30 studies used the asynchronous control to conduct critical statistical comparisons with the synchronous condition. Thus, the previous literature was not reviewed correctly, and the results of the preregistered analysis concerning condition-specific relationships deserve more careful consideration than by Lush et al. [8]

A critical idea in phenomenological control theory is that synchronous and asynchronous conditions differ in demand characteristics and implicit suggestions. Therefore, these conditions trigger different phenomenological control, leading to the observed differences in the RHI measures. However, this hypothesis is not supported by the results, nor does it fit with the past literature. If differences in implicit suggestions between the two conditions lead to different degrees of phenomenological control, as the authors theorized, this effect should be most pronounced in highly hypnotizable individuals. However, neither the findings of our analysis (Fig 1A-C) nor the preregistered analysis [7] support this prediction. Furthermore, if the condition-specific differences are related to demand characteristics, they should be affected by explicit suggestions. However, we observed no significant differences in RHI ratings among the three groups that received different suggestion instructions in our analysis (Fig 1D). These observations do not support the authors' theory but are well in line with the view that the $\mathrm{RHI}$ is a perceptual illusion, which should be resistant to suggestions, thoughts, and high-level conceptual knowledge.

A related concern is that the authors exaggerated the effect of demand characteristics in $\mathrm{RHI}$ paradigms and ignored the past literature that attempted to minimize and control it in various ways. For example, they did not consider previous studies that associated synchronyasynchrony differences in the RHI to the temporal window of multisensory integration. Such studies systematically manipulated the degree of asynchrony and found that the illusion gradually diminished as visual and tactile stimuli became increasingly temporally incongruent, and the RHI was abolished for asynchronies greater than 200-300 ms [9-12]. This precise temporal rule fits well with the temporal window of integration of multisensory cortical neurons [13], causal inference theories of multisensory integration [6,12,14], and the past body representation literature $[1,2,4,6,9-12,15,16]$. However, this precise temporal rule is not explained by phenomenological control theory because suggestions have been well 
controlled in studies using subtle experimental manipulations of asynchrony. Psychophysical approaches that better control for cognitive bias have been used in RHI studies [12].

In summary, the results from the study by Lush and colleagues [7] provide an incremental addition to the growing literature on individual differences in the RHI. However, they do not present evidence that fundamentally alters our understanding of the relationship between top-down control of experience and perceptual mechanisms.

\section{References}

1. Botvinick, M. and J. Cohen, Rubber hands 'feel' touch that eyes see. Nature, 1998. 391(6669): p. 756.

2. Tsakiris, M. and P. Haggard, The rubber hand illusion revisited: visuotactile integration and self-attribution. J Exp Psychol Hum Percept Perform, 2005. 31(1): p. 80-91.

3. Riemer, M., et al., The rubber hand universe: On the impact of methodological differences in the rubber hand illusion. Neurosci Biobehav Rev, 2019. 104: p. 268-280.

4. Tsakiris, M., My body in the brain: a neurocognitive model of body-ownership. Neuropsychologia, 2010. 48(3): p. 703-12.

5. Ehrsson, H.H., Multisensory processes in body ownership, in Multisensory Perception: From Laboratory to Clinic, K. Sathian and V.S. Ramachandran, Editors. 2020, Elsevier Inc./Academic Press: San Diego.

6. Samad, M., A.J. Chung, and L. Shams, Perception of body ownership is driven by Bayesian sensory inference. PLoS One, 2015. 10(2): p. e0117178.

7. Lush, P., et al., Trait phenomenological control predicts experience of mirror synaesthesia and the rubber hand illusion. Nat Commun, 2020. 11(1): p. 4853.

8. Lush, P., et al., Phenomenological control: response to imaginative suggestion predicts measures of mirror touch synaesthesia, vicarious pain and the rubber hand illusion. 2019: PsyArXiv preprints.

9. Shimada, S., K. Fukuda, and K. Hiraki, Rubber hand illusion under delayed visual feedback. PLoS One, 2009. 4(7): p. e6185.

10. Shimada, S., et al., Relationship between sensitivity to visuotactile temporal discrepancy and the rubber hand illusion. Neurosci Res, 2014. 85: p. 33-8.

11. Costantini, M., et al., Temporal limits on rubber hand illusion reflect individuals' temporal resolution in multisensory perception. Cognition, 2016. 157: p. 39-48.

12. Chancel, M. and H.H. Ehrsson, Which hand is mine? Discriminating body ownership perception in a two-alternative forced-choice task. Atten Percept Psychophys, 2020.

13. Avillac, M., S. Ben Hamed, and J.R. Duhamel, Multisensory integration in the ventral intraparietal area of the macaque monkey. J Neurosci, 2007. 27(8): p. 1922-32.

14. Kording, K.P., et al., Causal inference in multisensory perception. PLoS One, 2007. 2(9): p. e943.

15. Longo, M.R., et al., What is embodiment? A psychometric approach. Cognition, 2008. 107(3): p. 978-98.

16. Tsakiris, M., et al., Hands only illusion: multisensory integration elicits sense of ownership for body parts but not for non-corporeal objects. Exp Brain Res, 2010. 204(3): p. 343-352. 

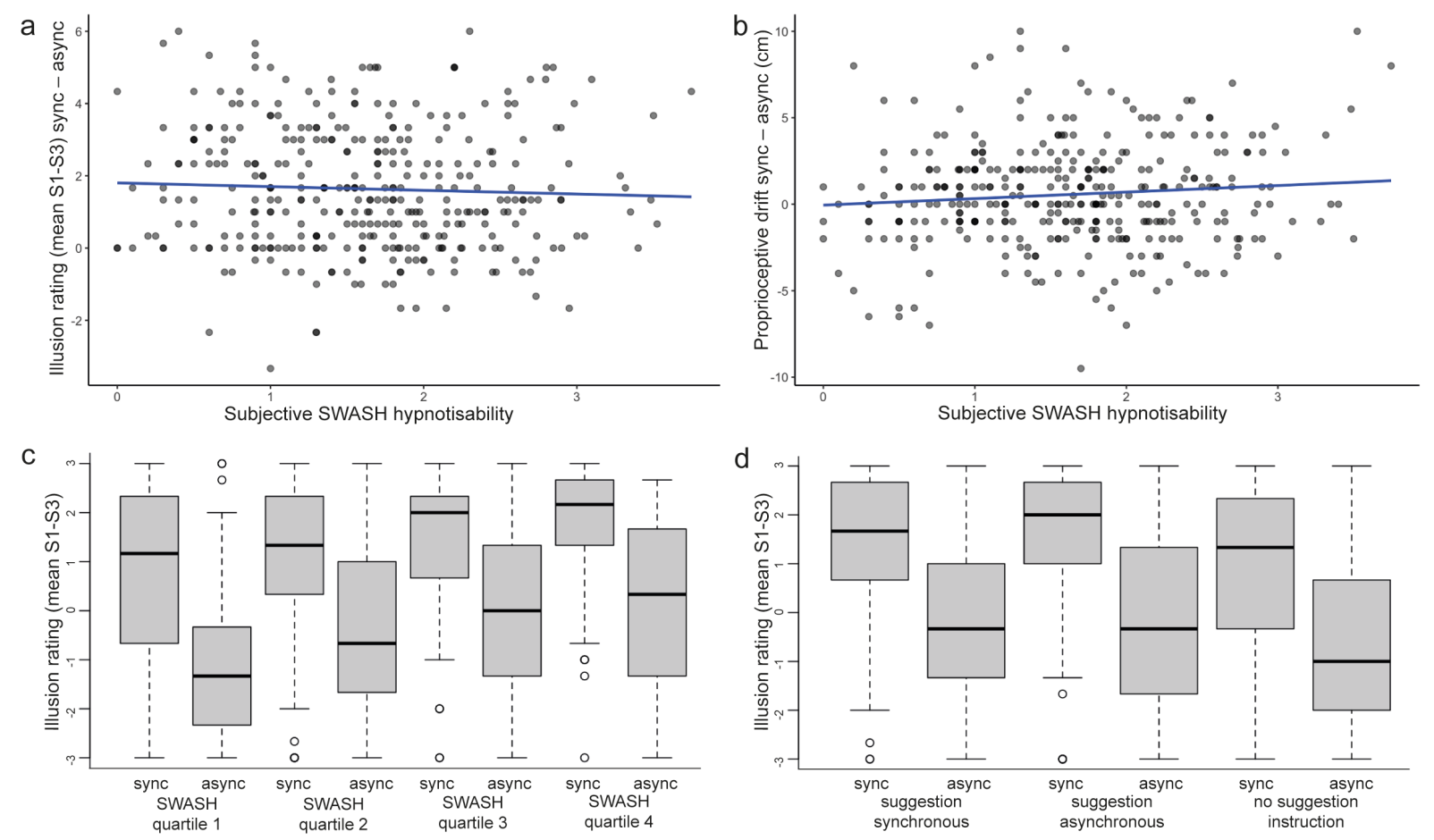

Fig 1. The rubber hand illusion (RHI) is resistant to hypnotic suggestibility. A. No significant relationship between trait hypnotic suggestibility (SWASH) and illusion ratings (mean of questionnaire statements S1 to S3 [7]) when comparing the synchronous (illusion) and asynchronous (control) conditions ( $\varrho=-0.06563398, p=0.2187,95 \% \mathrm{Cl}[-0.16885998,0.03901619], \mathrm{BF}_{01}=5.684709, \mathrm{n}=353$ ). B. Similarly, there was no significant relationship between SWASH and proprioceptive drift when contrasting the conditions $\left(\varrho=0.04728089, p=0.3758,95 \% \mathrm{Cl}[-0.05738520,0.15091893], \mathrm{BF}_{01}=\right.$ $1.656704, n=353)$. C. When dividing the participants into four quartiles based on the trait of hypnotic suggestibility (SWASH quartiles 1-4), the illusion is significantly induced in all groups, including the least hypnotically suggestible subjects (quartile 1 ). The differences in illusion ratings between the synchronous and asynchronous conditions were significant in all four quartiles $(1: V=2575.5, p<0.001$, $\mathrm{n}=88 ; 2: \mathrm{V}=2932, \mathrm{p}<0.001, \mathrm{n}=89 ; 3: \mathrm{V}=3198, \mathrm{p}<0.001, \mathrm{n}=92 ; 4: \mathrm{V}=2779.5, \mathrm{p}<0.001, \mathrm{n}=84)$, and the synchronous conditions were always rated significantly greater than zero $(1: V=2387.5, p=$ $0.0002283 ; 2: V=3258.5, p<0.001 ; 3: V=3724, p<0.001 ; 4: V=3271.5, p<0.001)$. Moreover, nonparametric one-way ANOVA (Kruskal-Wallis $\mathrm{H}$ test) on difference in illusion ratings between the synchronous and the asynchronous conditions in four quartiles was not significant $(\chi 2=2.9133, \mathrm{df}=3$, $p=0.4052$; a direct comparison between quartile 1 and quartile 4 was also non-significant: $W=3879.5$, $p=0.574)$. D Explicit suggestions before the experiment commenced did not influence the illusion since the difference in illusion rating between the synchronous and asynchronous condition was not significantly different in the three groups that were informed that they would experience the strongest illusion in the synchronous condition (suggestion synchronous; $n=114$ ), in the asynchronous condition (suggestion asynchronous; $\mathrm{n}=115$ ) or when receiving no information (no suggestion instruction; $n=124$ ) (suggestion sync vs suggestion async: $W=6382, p=0.7301$; suggestion sync vs no suggestion instruction: $W=7178, p=0.8361$; suggestion async vs no suggestion instruction: $W=7422.5, p=$ $0.5836)$. In all three groups, the illusion was significantly induced when contrasting the two conditions $(V=5438, p<0.001 ; V=4697, p<0.001 ; V=5169.5, p<0.001)$. The data from [7] are openly available and were analyzed with RStudio software, version 1.3.1056, and the BayesFactor software package, version 0.9.12-4.2. Nonparametric tests were used (Wilcoxon signed-rank test for paired comparisons, Mann-Whitney $U$ test for independent group comparisons) since the questionnaire data were ordinal and not normally distributed; two-tailed tests were performed. For the Bayes factor analysis, the default Cauchy priors were used. Sync: synchronous condition; Async: asynchronous condition; 
SWASH: Sussex-Waterloo Scale of Hypnotizability. The boxplots (in C and D) depict the data based on their median (thick black line) and quartiles (upper and lower ends of boxes). The vertical lines, i.e., the whiskers, indicate the minimum or maximum values within $1.5 \mathrm{x}$ the interquartile range above and below the upper and lower quartile. The circles denote outlier observations, the furthest being the minimum or maximum values in the data. 


\section{Supplementary Information}
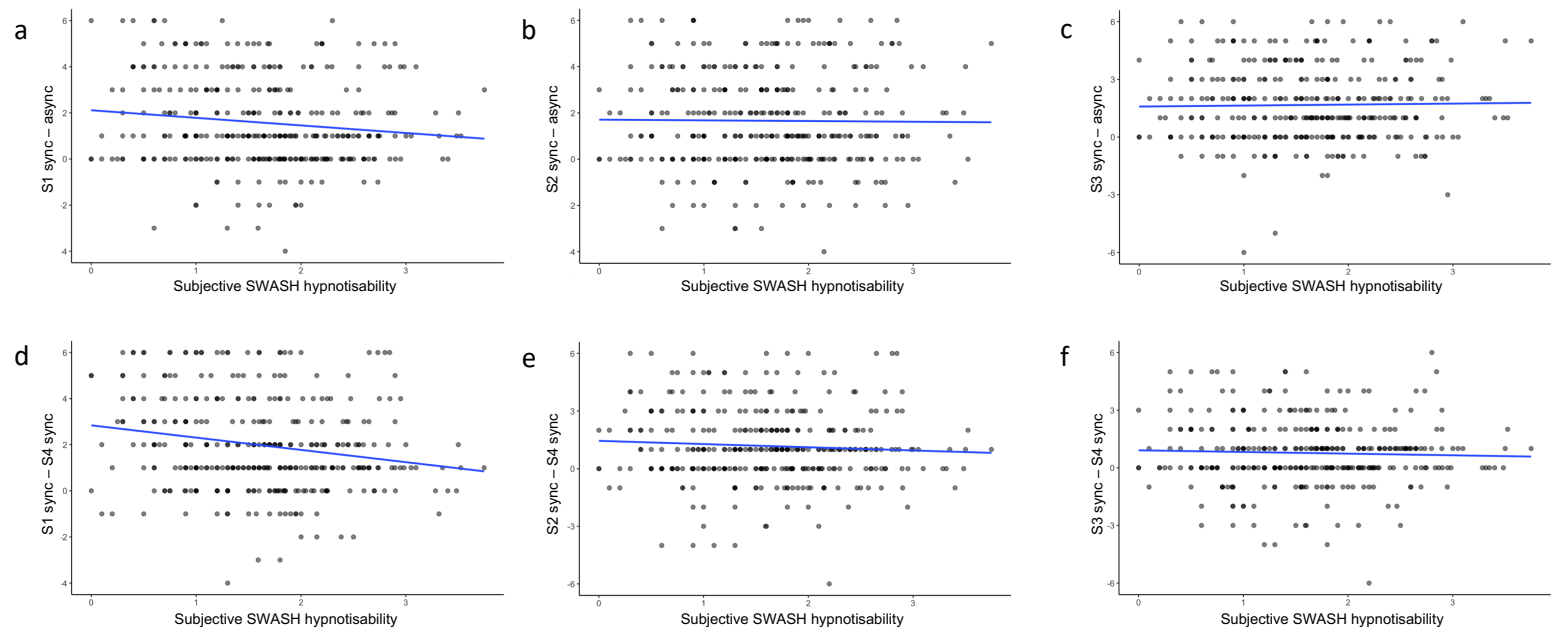

Suppl Fig 1. A. Significant negative relationship between trait hypnotic suggestibility (SWASH) and the ratings on the first illusion statement, referring to sensing touch on the rubber hand (questionnaire statement S1), when comparing the synchronous (illusion) and the asynchronous (control) conditions ( $e=-0.1366661, p=0.01015,95 \% \mathrm{Cl}[-0.23765859-0.03275039], \mathrm{BF} 01=0.442654, \mathrm{n}=353)$. That is, a negative relationship directly against the authors' (one-tailed) pre-registered hypothesis. B. No significant relationship between trait hypnotic suggestibility (SWASH) and the ratings on the second illusion statement, which relates to experiencing the touch one feels being caused by the touches one sees on the rubber hand (questionnaire statement S2), when comparing the synchronous (illusion) and the asynchronous (control) conditions $(e=-0.02160634, p=0.6858,95 \% \mathrm{Cl}[-$ $0.125705710 .08296364]$, BF01 $=7.880239, n=353$ ). C. No significant relationship between trait hypnotic suggestibility (SWASH) and ratings on the third illusion statement (questionnaire statement S3) that refers to the explicit feeling of ownership of the rubber hand when comparing the synchronous (illusion) and the asynchronous (control) conditions $(e=0.002540252, p=0.9621,95 \% \mathrm{Cl}[-$ $0.1018696320 .106894781]$, BF01 $=7.524658, n=353$ ). D. Significant negative relationship between trait hypnotic suggestibility (SWASH) and the rating difference score when comparing the illusion statement S1 to control statement S4 $(e=-0.1889739, p=0.0003638,95 \% \mathrm{Cl}[-0.28781962-$ $0.08614434], \mathrm{BF0}=0.008655026, \mathrm{n}=353$ ); that is a relationship that goes against the predication of the phenomenological control hypothesis. E. No significant relationship between trait hypnotic suggestibility (SWASH) and the rating difference score when comparing the illusion statement $\mathrm{S} 2$ to control statement S4 ( $e=-0.06295493, p=0.2388,95 \% \mathrm{Cl}[-0.166391170 .04185171], \mathrm{BF01}=3.83133$, $\mathrm{n}=353$ ). F. No significant relationship between trait hypnotic suggestibility (SWASH) and rating difference score when comparing the illusion statement S3 to control statement S4 ( $e=-0.0132043, p$ $=0.805,95 \% \mathrm{Cl}[-0.117573240 .09145315], \mathrm{BF} 01=6.257215, \mathrm{n}=353)$. 